Arq. Bras. Med. Vet. Zootec., v.66, n.1, p.109-115, 2014

\title{
Tranquilização de asininos com acepromazina associada ou não ao diazepam
}

\author{
[Tranquilization of donkeys using acepromazine or acepromazine/diazepam] \\ A.L. Araújo ${ }^{1}$, G.A.F. Souza ${ }^{1}$, P.I. Nóbrega Neto ${ }^{2}$, A.P. Souza ${ }^{2}$ \\ ${ }^{1}$ Aluna de pós-graduação - Centro de Saúde e Tecnologia Rural - Universidade Federal de \\ Campina Grande - CSTR-UFCG - Patos, PB \\ ${ }^{2}$ Centro de Saúde e Tecnologia Rural - Universidade Federal de Campina Grande - Patos, PB
}

\begin{abstract}
RESUMO
Avaliaram-se os efeitos da acepromazina isolada ou associada ao diazepam em asininos. Cinco asininos foram submetidos a dois protocolos anestésicos: os do grupo acepromazina (AC) receberam acepromazina, $0,1 \mathrm{mg} / \mathrm{kg} / \mathrm{IV}$, e os do grupo acepromazina-diazepam (ACD), acepromazina na mesma dose e via do AC, associada ao diazepam, $0,1 \mathrm{mg} / \mathrm{kg} / \mathrm{IV}$. Foram mensuradas as frequências cardíaca (FC) e respiratória (FR) e a temperatura retal (TR) e analisadas variáveis eletrocardiográficas, tranquilização, período de latência, início do prolapso peniano e grau de ataxia. A tranquilização iniciou-se aos 10,4 $\pm 0,9$ minutos nos asininos do $\mathrm{AC}$ e aos 4,8 $\pm 1,1$ nos do ACD. Ocorreu prolapso peniano aos $4,2 \pm 1,3 \mathrm{~min}$ no AC e aos $2,7 \pm 0,4$ no ACD. A FC elevou-se aos 15 e 30 min no AC. Não ocorreu variação significativa nas variáveis eletrocardiográficas e na temperatura retal. A FR diminuiu no AC a partir de 60min e no ACD a partir de 30min. A distância focinho-solo reduziu-se significativamente em ambos os grupos e nos momentos a partir de $15 \mathrm{~min}$. Concluiu-se que a acepromazina promove tranquilização discreta, e a adição do diazepam potencializa a tranquilização, diminui o período de latência e aumenta a ataxia.
\end{abstract}

Palavras-chave: jumento, fenotiazínico, benzodiazepínico, medicação pré-anestésica

\begin{abstract}
The effects of acepromazine isolated or associated with diazepam were evaluated in five donkeys were underwent in two anesthetic protocols, in the acepromazine group (AC), animals received acepromazine $(0.1 \mathrm{mg} / \mathrm{kg} / \mathrm{IV})$ and in acepromazine-diazepam group $(A C D)$, acepromazine at the same dose and route of $A C$, associated with diazepam $(0.1 \mathrm{mg} / \mathrm{kg} / \mathrm{IV})$. Heart frequency $(\mathrm{HR})$, respiratory frequency $(R F)$ and rectal temperature $(R T)$ were measured and electrocardiographic variables analyzed, in addition to tanquilization, latency, beginning of the penile prolapse and degree of ataxia. The tanquilization began at $10.4 \pm 0.9$ minutes (min) in the AC donkeys and $4.8 \pm 1.1$ in ACD. Penile prolapse occurred at $4.2 \pm 1.3$ minutes in $A C$ and $2.7 \pm 0.4$ in ACD. The HR increased to 15 and 30min. Electrocardiographic parameters and rectal temperature not varied significantly. $R F$ decreased from $A C$ in $60 \mathrm{~min}$ and $30 \mathrm{~min}$ from ACD. The muzzle-to-ground distance reduced significantly in both groups and at times from 15min. It was concluded that the acepromazine promotes discreet tanquilization and the adition of diazepam potentiates the tranquilization, decreases the latency period and increases ataxia caused by acepromazine.
\end{abstract}

Keywords: donkey, phenothiazine, benzodiazepine, premedication

\section{INTRODUÇÃO}

A capacidade de sobrevivência de asininos em ambientes adversos e de apresentarem maior resistência ao trabalho associada a peculiaridades anatomofisiológicas da espécie demonstram que há diferenças entre os equídeos e que os asininos precisam de assistência técnica especializada que garanta maior segurança na execução de procedimentos clínicos e cirúrgicos. Vários fármacos que proporcionam contenção farmacológica em equinos, na maioria das vezes, não proveem o mesmo aos asininos.

Recebido em 15 de abril de 2013

Aceito em 25 de junho de 2013

E-mail: araujonascal@gmail.com 
Há muitos relatos da eficácia da acepromazina em equinos como agente tranquilizante ou em associações anestésicas (Marroum et al., 1994; Adair III et al., 1997) ao promover sinais característicos de sua ação, como ptose palpebral, discreta protusão da membrana nictitante, prolapso peniano e abaixamento da cabeça (Booth e McDonald, 1992). Contudo poucos são os trabalhos direcionados a asininos. Matthews e Van Dijk (2004) observaram que, diferentemente de mulas, os jumentos são em geral bem sedados e tranquilizados quando se usam doses de fármacos similares às usadas em cavalos.

Doses diversas de tranquilizantes são citadas na literatura para asininos e muares sem, contudo, relatar seus efeitos. Segundo Matthews e Taylor (2000), a administração de acepromazina na dose de $0,04 \mathrm{mg} / \mathrm{kg}$, pela via intravenosa (IV), promove tranquilização satisfatória. Matthews e Van Dijk (2004) descreveram que esse tranquilizante, na dose de $0,1 \mathrm{mg} / \mathrm{kg}$, via IV ou intramuscular (IM), ou butorfanol, na dose de $0,02-0,04 \mathrm{mg} / \mathrm{kg}, \mathrm{IV}$, combinado à xilazina, na dose de 0,6 - 1,0mg/kg, IV ou IM, foram relativamente suficientes, tanto para procedimentos em estação - com anestesia local -, ou antes de anestesia geral.

Outro fármaco empregado em equinos com intuito de miorrelaxamento ou sinergismo entre fármacos é o diazepam (Gross, 2003). Neles, esse benzodiazepínico, administrado na dose de $0,2 \mathrm{mg} / \mathrm{kg}$, via IV, promove olhar fixo, tremores musculares da cabeça, pescoço e tórax, ataxia, movimentação de um lado ao outro, inclinação contra a cerca ou cruzamento dos membros pélvicos (Muir et al., 1982) e ptose palpebral (Korttila e Linnoila, 1975).

Em asininos é recomendada a adição de benzodiazepínicos no protocolo de indução anestésica com intuito de promover relaxamento muscular e melhor sedação (Matthews e Van Dijk, 2004). Matthews e Taylor (2000) afirmaram que, em jumentos, a anestesia injetável é mais satisfatória quando butorfanol, na dose de $0,04 \mathrm{mg} / \mathrm{kg}$ IV, ou diazepam, $0,03 \mathrm{mg} / \mathrm{kg} \mathrm{IV}$, são combinados com xilazina ou detomidina para aumentar a sedação produzida.
Diante da necessidade de obter mais informações sobre protocolos usados para conteção de asininos, objetivou-se com este estudo avaliar a ação tranquilizante da acepromazina e o sinergismo decorrente da associação da acepromazina com o diazepam, e os efeitos dos mesmos sobre alguns parâmetros fisiológicos de asininos.

\section{MATERIAL E MÉTODOS}

O presente estudo foi aprovado pelo Comitê de Ética e Pesquisa do Centro de Saúde e Tecnologia Rural da Universidade Federal de Campina Grande, Campus de Patos, Paraíba, sob o protocolo de $n^{\circ} 85 / 2008$.

Foram utilizados cinco asininos adultos, sem raça definida, machos, com média de peso de $99 \pm 17 \mathrm{~kg}$, clinicamente sadios, pertencentes ao Serviço de Recolhimento de Animais da Prefeitura de Patos, PB. Durante todo o período experimental, os animais foram mantidos nos currais do Hospital Veterinário escola, onde receberam feno de capim-Tifton (Cynodon dactylon) e água à vontade. Foi respeitado um período de duas semanas para adaptação dos animais às instalações, antes que o experimento tivesse início.

Cada animal participou, de forma aleatória, de ambos os grupos experimentais, previamente designados de grupo acepromazina (AC) e grupo acepromazina-diazepam (ACD), respeitando-se o intervalo de 15 dias entre os tratamentos. Após jejum alimentar de 12 horas, cada animal foi pesado e levado à sala de experimentação. Os asininos do AC foram medicados com acepromazina (Acepran 1\% - Lab. Univet S.A. Indústria Veterinária - São Paulo, Brasil), na dose de $0,1 \mathrm{mg} / \mathrm{kg}$, pela via IV. Os do ACD receberam acepromazina, na dose de $0,1 \mathrm{mg} / \mathrm{kg}$, associada ao diazepam (Compaz 0,5\% - Cristália Produtos Quim. e Farm. Ltda. - Itapira, SP, Brasil), na mesma dose e via. Após a administração dos fármacos, os animais foram deixados à vontade dentro da sala de experimentação, até o final do período experimental, após o qual foram levados de volta ao respectivo curral. 
Para avaliação da qualidade da tranquilização, mensuraram-se: o período de latência (tempo decorrido entre o final da administração dos fármacos e o início da tranquilização, caracterizado pelo começo da ptose palpebral); a intensidade da tranquilização (aferida pela distância entre o focinho do animal e o solo); o momento de início e a duração do prolapso peniano; o grau de ataxia apresentado (por meio do emprego da seguinte escala: 0 - ataxia ausente; 1 - ataxia moderada, consegue deambular; 2 - ataxia grave, com risco de decúbito).

Em ambos os grupos, mensurou-se frequência cardíaca (FC) (bat/min), calculada a partir do intervalo R-R obtido no eletrocardiógrafo computadorizado (TEB - mod. ECGPC software versão 1.10 - São Paulo, Brasil), usado também para registro do eletrocardiograma (ECG) em derivação DII, sensibilidade normal (N) e velocidade de $25 \mathrm{~mm} /$ segundo, observando os valores referentes à duração e amplitude da onda P (Pms e PmV), duração do complexo QRS (QRSms), amplitude da onda R (RmV) e onda $\mathrm{T}(\mathrm{TmV})$, e os intervalos em milissegundos (ms) entre as ondas P e R (PR), Q e T (QT) e entre duas ondas $\mathrm{R}$ subsequentes (RR). Para a realização da eletrocardiografia, os eletrodos adesivos (Eletrodo adesivo para eletrocardiografia modelo 2223 - 3M Brasil Ltda.) foram posicionados um na região cervical e os outros dois nas áreas dorsal e ventral da região torácica esquerda (configuração pescoçocernelha). Aferiu-se ainda a frequência respiratória (FR) (mov/min), por inspeção dos movimentos torácicos durante um minuto, e a temperatura retal (TR) $\left({ }^{\circ} \mathrm{C}\right)$, empregando-se um termômetro clínico digital. Todas as variáveis foram mensuradas imediatamente antes da administração do(s) fármaco(s) (T0) e a cada 15 minutos após a administração, T15, T30 ... T120, durante 120 minutos, momentos nos quais realizou-se também a avaliação da intensidade da tranquilização.

A análise estatística foi efetuada em microcomputador, empregando o programa Graphpad Instat (GraphPad Software, Inc. - San Diego, California, USA). Os dados paramétricos foram analisados com o emprego da análise de variância para amostras repetidas, e a comparação entre os momentos e entre os grupos foi realizada pelo teste de SNK. Os dados referentes ao período de latência, ao início do prolapso peniano e ao grau de ataxia, foram avaliados empregando o teste $t$. Todos os testes foram aplicados para 5\% de significância.

\section{RESULTADOS E DISCUSSÃO}

Os animais que receberam apenas acepromazina apresentaram sinais de tranquilização discreta, com abaixamento de cabeça, ptose palpebral e indiferença ao ambiente, aos 10,4 $\pm 0,9$ minutos, enquanto nos do grupo ACD tais sinais surgiram significativamente mais precoce e intensamente, aos 4,8 $\pm 1,1$ minutos.

A administração da acepromazina isoladamente por certo não forneceu tranquilização similar à promovida pela mesma nos equinos (Booth e McDonald, 1992), sugerindo maior resistência do asinino ao fármaco. No entanto, Matthews e Taylor (2000) afirmaram que a dose de $0,04 \mathrm{mg} / \mathrm{kg}$ promove satisfatória tranquilização em jumentos e mulas. Essa diferença de resultados pode ser justificada, possivelmente, pelas variações individuais, como exposto pela experiência clínica de Matthews e Van Dijk (2004). Contudo, pode-se inferir que a tranquilização promovida pela acepromazina é suficiente para permitir exploração física de asininos, como também atenuar os estímulos externos.

A associação do diazepam ao fenotiazínico diminuiu o período de latência deste e potencializou sua ação tranquilizante, eventos já constatados por Korttila e Linnoila (1975) e Gross (2003) em outras espécies. Tais efeitos são desejados nos casos de procedimentos mais invasivos, nos quais é esperado mínimo estresse e desconforto ao animal, e são extremamente vantajosos em se tratando de asininos, pois os animais tornam-se indiferentes ao meio ambiente que os cerca e, portanto, mais cooperativos quando da contenção.

Dois animais do grupo ACD apresentaram tremores musculares nos membros e pescoço, iniciados cerca de 40 segundos após a administração dos fármacos e durando em média 10 minutos. Os tremores, sugestivos de miorrelaxamento, decorreram do efeito abrupto causado pelo diazepam sobre a musculatura esquelética (Gross, 2003). Esse miorrelaxamento, apesar de interessante do ponto 
de vista cirúrgico, é inconveniente quando se trabalha com o animal na posição quadrupedal, pois aumenta a ataxia e o risco de decúbito. Assim, a associação acepromazina-diazepam seria mais interessante no protocolo de indução da anestesia geral ou dissociativa (Matthews e Van Dijk, 2004), buscando relaxamento muscular e melhor sedação.

Como esperado, todos os animais apresentaram prolapso peniano, visto que a acepromazina causa relaxamento do músculo retrator do pênis. No grupo ACD, constatou-se significativa diminuição no tempo decorrido até o início do prolapso $(2,7 \pm 0,4$ minutos) comparado ao $\mathrm{AC}$ (4,2 $\pm 1,3$ minutos), confirmando a potencialização do diazepam sobre os efeitos do fenotiazínico. Todos os animais ainda apresentavam prolapso peniano ao final do período experimental, não sendo constatado nenhum caso de priapismo ou prolapso persistente, constatação relevante para o uso da acepromazina em asininos na dose empregada.

A tranquilização avaliada segundo a altura focinho-solo foi diferente entre tempos a partir do T15 nos dois grupos, e não diferiu entre grupos (Fig. 1). No grupo AC, constatou-se redução média de $27 \%$,e no $\mathrm{ACD}, 33,5 \%$, em relação ao valor inicial, permanecendo baixa por todo o período experimental. A não diferenciação significativa dentre os grupos difere da observação clínica, a qual demonstrou ser mais expressiva no ACD. Ao final do período experimental, os animais ainda apresentaram sinais de tranquilização.

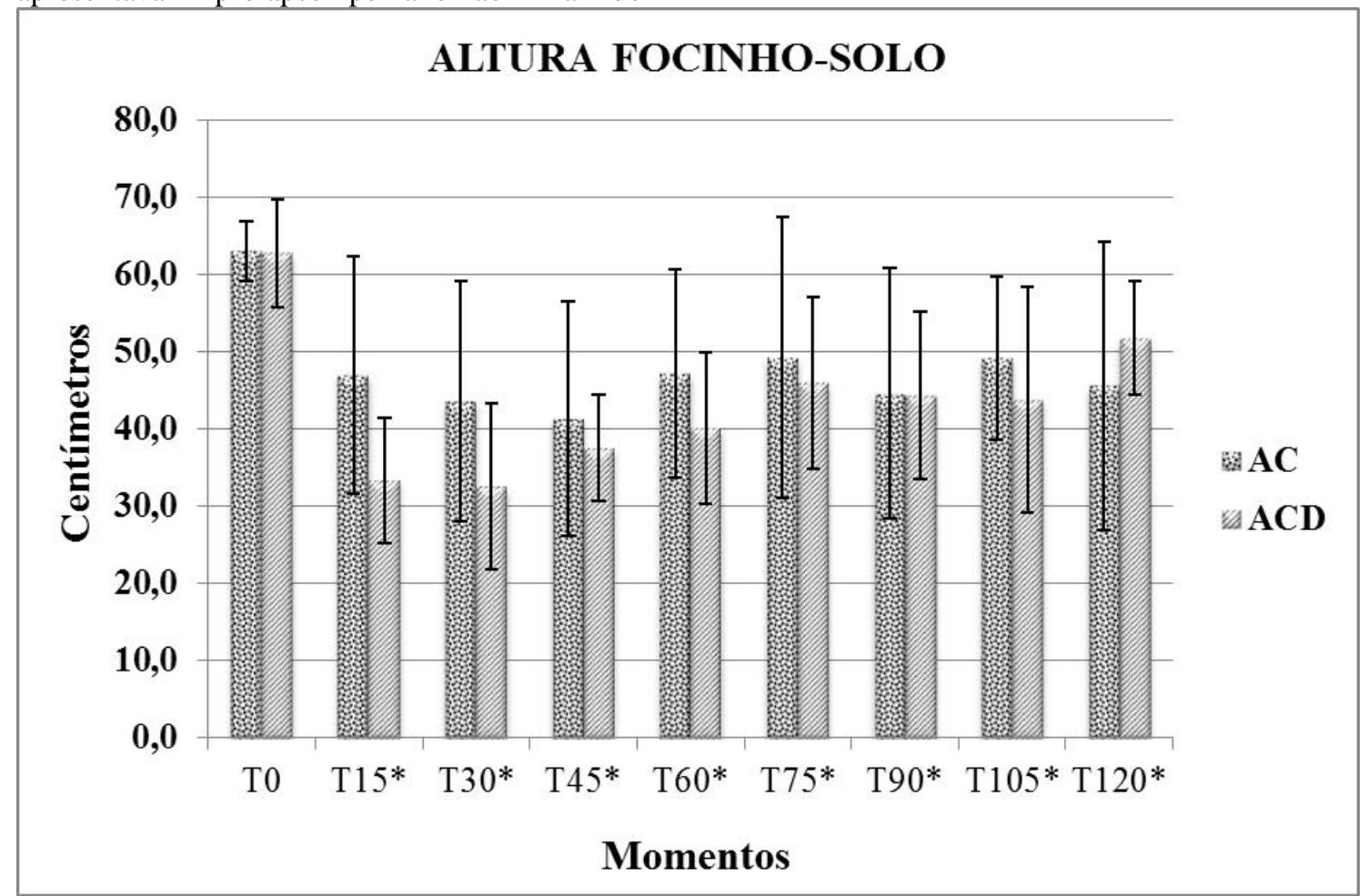

*Estatisticamente diferente de T0 $(\mathrm{P}<0,05)$.

Figura 1. Variação dos valores médios e desvios padrão da altura focinho-solo (cm), em diferentes momentos, de asininos tranquilizados com acepromazina (AC) e acepromazina associada ao diazepam $(\mathrm{ACD})(\mathrm{n}=5)$.

Nenhum dos animais do grupo AC apresentou ataxia, enquanto nos do ACD, 15 minutos após a administração dos fármacos, esta foi classificada como moderada e significativamente maior,
$1,6 \pm 0,5$, que a apresentada pelos animais do grupo AC, 0 - ausente. Do T30 em diante, esse grau de ataxia foi classificado como $0,6 \pm 0,5$ no grupo ACD, não mais diferindo do grupo AC. A 
maior ataxia apresentada pelos animais do grupo ACD, nos primeiros 15 minutos após a administração dos fármacos, deve-se o efeito do diazepam sobre a musculatura dos membros e pescoço. Provavelmente, a redistribuição do diazepam fez com que os níveis plasmáticos do mesmo diminuíssem, o que reduziu o relaxamento muscular e a ataxia, a partir do momento T30. Tallman et al. (1980) também descreveram efeitos como ataxia após o uso do diazepam.
Ocorreu aumento significativo da FC (Tab. 1) em T15 e T30 no grupo AC, o qual foi atribuído ao fato de a acepromazina reduzir a resistência vascular sistêmica e a pressão arterial, desencadeando aumento compensatório nos batimentos cardíacos que resulta, consequentemente, no aumento inicial do débito cardíaco (Muir e Mason, 1993). O diazepam não influenciou essa variável, conforme citado por Kanto e Klotz (1982).

Tabela 1. Valores médios ( $\bar{x}$ ) e desvios padrão (s) das frequências cardíaca (FC) e respiratória (FR) e de temperatura retal (TR), em asininos tranquilizados com acepromazina (AC) e acepromazina associada ao diazepam $(\mathrm{ACD})(\mathrm{n}=5)$

\begin{tabular}{|c|c|c|c|c|c|c|c|c|c|c|c|}
\hline \multirow{2}{*}{ Variável } & \multirow{2}{*}{ Grupo } & & \multicolumn{9}{|c|}{ Momentos (minutos após administração dos fármacos) } \\
\hline & & & T0 & T15 & T30 & $\mathrm{T} 45$ & T60 & $\mathrm{T} 75$ & T90 & $\mathrm{T} 105$ & $\mathrm{~T} 120$ \\
\hline \multirow{4}{*}{$\begin{array}{c}\mathrm{FC} \\
\text { (bat/min) }\end{array}$} & \multirow{2}{*}{$\mathrm{AC}$} & $\bar{x}$ & 30 & $39 *$ & $39 *$ & 35 & 34 & 33 & 32 & 32 & 30 \\
\hline & & $\mathrm{s}$ & 2,6 & 7,8 & 9,2 & 7,2 & 7,2 & 4,2 & 4,6 & 4,1 & 4,9 \\
\hline & \multirow[t]{2}{*}{$\mathrm{ACD}$} & $\bar{x}$ & 31 & 41 & 39 & 36 & 32 & 34 & 35 & 31,0 & 31,0 \\
\hline & & $\mathrm{s}$ & 5,0 & 11,2 & 5,1 & 4,0 & 4,6 & 8,7 & 7,8 & 5,0 & 4,3 \\
\hline \multirow{3}{*}{$\begin{array}{c}\text { FR } \\
(\mathrm{mov} / \mathrm{min})\end{array}$} & \multirow[t]{2}{*}{$\mathrm{AC}$} & $\bar{x}$ & 18 & 14 & 15 & 14 & $12 *$ & $12 *$ & $12 *$ & $13^{*}$ & $12 *$ \\
\hline & & s & 7,7 & 3,5 & 3,3 & 3,7 & 2,6 & 2,6 & 2,2 & 2,3 & 0,9 \\
\hline & \multirow[t]{2}{*}{ ACD } & $\bar{x}$ & 16 & 14 & $12 *$ & $12 *$ & $12 *$ & $12 *$ & $11 *$ & $11 *$ & $11 *$ \\
\hline \multirow{5}{*}{$\begin{array}{l}\text { TR } \\
\left({ }^{\circ} \mathrm{C}\right)\end{array}$} & & $\mathrm{s}$ & 1,7 & 3,3 & 1,7 & 1,7 & 1,4 & 2,2 & 1,1 & 1,1 & 1,1 \\
\hline & \multirow[t]{2}{*}{$\mathrm{AC}$} & $\bar{x}$ & 36,1 & 36,4 & 36,5 & 36,4 & 36,4 & 36,3 & 36,3 & 36,3 & 36,3 \\
\hline & & $\mathrm{s}$ & 0,7 & 0,9 & 0,5 & 0,6 & 0,6 & 0,6 & 0,8 & 0,8 & 0,9 \\
\hline & \multirow[t]{2}{*}{$\mathrm{ACD}$} & $x$ & 36,1 & 36,2 & 36,2 & 36,1 & 36,1 & 36,1 & 36,2 & 36,1 & 36,0 \\
\hline & & $\mathrm{S}$ & 1,0 & 0,9 & 0,8 & 0,8 & 0,8 & 0,8 & 0,8 & 0,8 & 0,8 \\
\hline
\end{tabular}

*Estatisticamente diferente de T0 $(\mathrm{P}<0,05)$

As variáveis eletrocardiográficas (Tab. 2) não variaram entre os momentos ou entre os grupos. Essa ausência de alterações confirma a inocuidade da acepromazina e do diazepam sobre a eletrofisiologia cardíaca, referida por Kanto e Klotz (1982) e Geiser (1990).

A frequência respiratória (Tab. 1) diminuiu significativamente no grupo AC a partir de T60, e no grupo ACD, a partir de T30, sem diferenças intergrupos. Esse decréscimo possivelmente deveu-se à redução da sensibilidade dos quimiorreceptores ao dióxido de carbono (Cortopassi e Fantoni, 2009), inabilitando o sistema nervoso autônomo da percepção do aumento de $\mathrm{CO}_{2}$, causada pela acepromazina. A rapidez na diminuição na $\mathrm{FR}$ no grupo ACD é condizente com a potencialização do benzodiazepínico sobre a acepromazina (Gross,
2003), antecipando seus efeitos. Essa diminuição da FR não caracteriza uma ocorrência preocupante em animais hígidos.

A TR não diferiu entre os momentos e entre grupos (Tab. 1). Todos os animais apresentaram, já no momento basal (T0), temperatura corpórea abaixo do limite considerado fisiológico descrito por Massone (2008). De qualquer forma, não foi detectada nenhuma influência dos fármacos administrados sobre essa variável, diferentemente do citado por Arena et al. (2009), quanto ao efeito da acepromazina na redução da temperatura corporal, ao relatar que esse fenotiazínico promove hipotermia pela depleção das reservas de dopamina no centro termorregulador, além da vasodilatação periférica. 
Tabela 2. Valores médios $(\bar{x})$ e desvios padrão (s) das variáveis eletrocardiográficas (Pms, PmV, PRms, QRSms, RmV, QT, e TmV), em asininos tranquilizados com acepromazina (AC) e associação acepromazina com diazepam (ACD) $(\mathrm{n}=5)$

\begin{tabular}{|c|c|c|c|c|c|c|c|c|c|c|c|}
\hline \multirow{2}{*}{\multicolumn{2}{|c|}{$\begin{array}{c}\text { Variável/ } \\
\text { Grupo }\end{array}$}} & & \multicolumn{9}{|c|}{ Momentos (minutos após administração dos fármacos) } \\
\hline & & & T0 & T15 & $\mathrm{T} 30$ & T45 & T60 & T75 & T90 & T105 & T120 \\
\hline \multirow{4}{*}{$\begin{array}{c}\mathrm{P} \\
(\mathrm{ms})\end{array}$} & \multirow{2}{*}{$\mathrm{AC}$} & $\bar{x}$ & 110,6 & 103,4 & 100,6 & 102,0 & 96,6 & 99,2 & 98,0 & 100,6 & 96,8 \\
\hline & & $\mathrm{s}$ & 12,3 & 14,0 & 13,8 & 8,4 & 13,1 & 19,1 & 9,9 & 11,9 & 11 \\
\hline & \multirow{2}{*}{ ACD } & $\bar{x}$ & 104,6 & 96,6 & 98,0 & 102,0 & 104,8 & 104,2 & 105,4 & 106,2 & 105,2 \\
\hline & & $\mathrm{s}$ & 12,1 & 18,1 & 12,4 & 4,9 & 11,6 & 9,4 & 12,7 & 12,8 & 16,7 \\
\hline \multirow{4}{*}{$\begin{array}{c}\mathrm{P} \\
(\mathrm{mV})\end{array}$} & \multirow{2}{*}{$\mathrm{AC}$} & $\bar{x}$ & $-0,38$ & $-0,34$ & $-0,34$ & $-0,35$ & $-0,35$ & $-0,33$ & $-0,36$ & $-0,35$ & $-0,36$ \\
\hline & & $\mathrm{s}$ & 0,14 & 0,12 & 0,13 & 0,12 & 0,13 & 0,11 & 0,16 & 0,15 & 0,16 \\
\hline & \multirow{2}{*}{$\mathrm{ACD}$} & $\bar{x}$ & $-0,39$ & $-0,36$ & $-0,36$ & $-0,36$ & $-0,36$ & $-0,35$ & $-0,37$ & $-0,38$ & $-0,37$ \\
\hline & & $\mathrm{s}$ & 0,12 & 0,06 & 0,07 & 0,10 & 0,08 & 0,08 & 0,08 & 0,10 & 0,10 \\
\hline \multirow{4}{*}{$\begin{array}{l}\mathrm{PR} \\
(\mathrm{ms})\end{array}$} & \multirow{2}{*}{$\mathrm{AC}$} & $\bar{x}$ & 228,6 & 231,2 & 237,2 & 237,0 & 238,6 & 239,4 & 233,4 & 242,0 & 237,6 \\
\hline & & $\mathrm{s}$ & 20,5 & 34,7 & 34,6 & 33,6 & 29,3 & 35,9 & 32,1 & 31,0 & 40,5 \\
\hline & \multirow{2}{*}{$\mathrm{ACD}$} & $\bar{x}$ & 230,0 & 218,6 & 221,2 & 232,6 & 239,8 & 232,6 & 219,2 & 224,6 & 215,8 \\
\hline & & $\mathrm{s}$ & 15,5 & 54,0 & 35,7 & 30,5 & 34,8 & 27,5 & 23,8 & 10,2 & 37,5 \\
\hline \multirow{4}{*}{$\begin{array}{l}\text { QRS } \\
(\mathrm{ms})\end{array}$} & \multirow{2}{*}{$\mathrm{AC}$} & $\bar{x}$ & 116,0 & 118,6 & 122,8 & 111,8 & 114,2 & 118,0 & 125,4 & 114,0 & 114,6 \\
\hline & & $\mathrm{s}$ & 10,4 & 7,7 & 17,9 & 11,7 & 17,9 & 7,7 & 5,1 & 7,7 & 9,2 \\
\hline & \multirow{2}{*}{ ACD } & $\bar{x}$ & 133,4 & 125,0 & 127,4 & 126,8 & 126,0 & 127,4 & 129,2 & 127,6 & 125,4 \\
\hline & & $\mathrm{s}$ & 15,2 & 4,5 & 13,5 & 12,3 & 11,8 & 7,6 & 8,9 & 1,3 & 12,1 \\
\hline \multirow{4}{*}{$\underset{(\mathrm{mV})}{\mathrm{R}}$} & \multirow{2}{*}{$\mathrm{AC}$} & $\bar{x}$ & 1,49 & 1,68 & 1,50 & 1,48 & 1,48 & 1,46 & 1,49 & 1,59 & 1,49 \\
\hline & & $\mathrm{s}$ & 0,43 & 0,62 & 0,44 & 0,46 & 0,45 & 0,42 & 0,42 & 0,52 & 0,47 \\
\hline & \multirow{2}{*}{$\mathrm{ACD}$} & $\bar{x}$ & 1,44 & 1,45 & 1,53 & 1,43 & 1,51 & 1,39 & 1,55 & 1,41 & 1,47 \\
\hline & & $\mathrm{s}$ & 0,36 & 0,27 & 0,27 & 0,20 & 0,26 & 0,26 & 0,26 & 0,26 & 0,32 \\
\hline \multirow{4}{*}{ QT } & \multirow{2}{*}{$\mathrm{AC}$} & $\bar{x}$ & 528,6 & 544,8 & 555,4 & 566,0 & 558,0 & 562,4 & 575,4 & 564,8 & 563,0 \\
\hline & & $\mathrm{s}$ & 39,1 & 39,0 & 27,8 & 15,8 & 16,6 & 9,3 & 21,0 & 19,6 & 21,9 \\
\hline & \multirow{2}{*}{$\mathrm{ACD}$} & $\bar{x}$ & 587,2 & 547,8 & 573,2 & 586,6 & 597,4 & 586,6 & 602,0 & 592,0 & 599,2 \\
\hline & & $\mathrm{s}$ & 41,0 & 72,0 & 56,4 & 37,0 & 41,0 & 51,5 & 54,9 & 44,2 & 55,3 \\
\hline \multirow{4}{*}{$\begin{array}{c}\mathrm{T} \\
(\mathrm{mV})\end{array}$} & \multirow{2}{*}{$\mathrm{AC}$} & $\bar{x}$ & 0,39 & 0,17 & 0,13 & 0,13 & 0,16 & 0,22 & 0,22 & 0,14 & 0,12 \\
\hline & & $\mathrm{s}$ & 0,55 & 0,54 & 0,61 & 0,55 & 0,58 & 0,54 & 0,54 & 0,59 & 0,55 \\
\hline & \multirow{2}{*}{$\mathrm{ACD}$} & $\bar{x}$ & 0,20 & $-0,26$ & $-0,11$ & 0,00 & 0,08 & 0,19 & 0,27 & 0,27 & 0,25 \\
\hline & & $\mathrm{s}$ & 0,86 & 0,37 & 0,54 & 0,53 & 0,65 & 0,83 & 0,67 & 0,67 & 0,63 \\
\hline
\end{tabular}

\section{CONCLUSÕES}

Evidenciou-se que a dose empregada da acepromazina promove tranquilização em asininos, a qual possibilita manipulação não invasiva. O emprego da associação acepromazina com diazepam potencializou o efeito tranquilizante do fenotiazínico, e os fármacos utilizados não alteram as variáveis fisiológicas estudadas na espécie.

\section{REFERÊNCIAS}

ADAIR III, H.S.; SCHMIDHAMMER, J.L.; GOBLE, D.O. et al. Effects of acepromazine maleate, isoxsuprine hydrochloride and prazosin hydrochloride on laminar microclrculatory blood flow in healthy horses. J. Equine Vet. Sci., v.17, p.599-603, 1997.

ARENA, G.; BOTELHO, A.; EVARISTO, B. et al. Fenotiazínicos: usos, efeitos e toxicidade em animais de grande e pequeno porte. Rev. Cient. Elet. Med. Vet., v.13, 2009. 
BOOTH, N.H.; McDONALD, L.E. Analgésicos não narcóticos. In:__. Farmacologia e terapêutica em veterinária. 6.ed. Rio de Janeiro: Guanabara Koogan, 1992. Cap.16. p.262-288.

CORTOPASSI, S.R.G.; FANTONI, D.T. Medicação Pré-anestésica. In: __. Anestesia em cães e gatos, São Paulo: Roca. 2009. Cap. 13, p.215-227.

GEISER, A.D. Chemical restraint and analgesia in the horse. Vet. Clin. N. Am-Equine., v.6, p.495-512, 1990.

GROSS, M.E. Tranquilizantes, agonistas $\alpha 2$ adrenérgicos e agentes relacionados. In: ADAMS, $\mathrm{H}$. R. Farmacologia e Terapêutica em Veterinária. 8. ed. Rio de Janeiro: Guanabara Koogan, 2003. Cap.14, p.249-274.

KANTO, J.; KLOTZ, U. Intravenous benzodiazepines as anaesthenic agents: Pharmacokinetics and clinical consequences. Acta. Anaesthesiol. Scand., v.26, p.554$569,1982$.

KORTTILA, K.; LINNOILA, M. Recovery and Skills Related to Driving after Intravenous Sedation: Doseresponse Relationship with Diazepam. Br. J. Anaesth., v.47. p.457-463, 1975.

MARROUM, P.J.; WEBB, A.I.; AESCHBACHER, G. et al. Pharmacokinetics and pharmacodinamics of acepromazine in horses. Am. J. Vet. Res., v.55, p.14281433, 1994.
MASSONE, F. Anestesiologia veterinária farmacologia e técnicas: texto e Atlas. 5.ed. Rio de Janeiro: Guanabara Koogan, 2008. 548p.

MATTHEWS, N.S.; TAYLOR, T.S. Anesthetic Management of Donkeys and Mules. In: STEFFEY E.P. (Ed.) Recent Advances in Anesthetic Management of Large Domestic Animals. Publisher: International Veterinary Information Service, July, 2000. Disponível em: $<$ http//:www.ivis.org/advances/Steffey_Anesthesia/ma thews_donkeys/ivis.pdf $>$. Acessado em: 20 ago. 2011.

MATTHEWS, N.S.; VAN DIJK, P. Anesthesia and Analgesia for Donkeys. In: MATTHEWS, N.S.; TAYLOR, T.S. (Ed.) Veterinary Care of Donkeys. Publisher: International Veterinary Information Service, Ithaca, New York, USA. Sep. 2004. Disponível em: 〈http//: www.ivis.org>. Acessado em: 21 ago. 2011.

MUIR, W.W.; MASON, D.E. Effects of diazepam, acepromazine, detomidine and xilazine on thiamilal anesthesia in horse J. Am. Vet. Med. Assoc., v.203, p.1031-1038, 1993.

MUIR, W.W.; SAMS, R.A.; HUFFMAN, R.H. et al. Pharmacodynamic and pharmacokinetic properties of diazepam in horses. Am. J. Vet. Res., v.43, p.17561768, 1982.

TALLMAN, J.F.; PAUL, S.M.; SKOLNICK, P. et al. Receptors for the age of anxiety: pharmacology of the benzodiazepines. Science, v.207, p.274-281, 1980. 